\title{
Estimation of the moisture content based on a thermal analysis method
}

\author{
Case of the sand \\ [ Chauchois Alexis, Derbal Radhouan, Defer Didier, Brachelet Franck ]
}

\begin{abstract}
A thermal analysis method based on inverse heat transfer is presented to estimate moisture content. The inverse method is based on experimental and numerical studies. A sand multilayer at different moisture contents is thermally excited. Thermocouple probes are placed at different positions in the thickness, and at the extremities of the multilayer, and record temperature variations during a test. A numerical heat transfer model associated with a parametric estimation algorithm allows layers' moisture contents identification. Results are obtained thanks to previous tests that provide empirical relationships between the thermophysical properties and the moisture content.
\end{abstract}

Keywords: Moisture content; Inverse heat transfer; Thermal characterization; Thermophysical properties.

\section{Introduction}

In the seventies oil crisis appeared. Furthermore, more recently earth has suffered from resource depletion, and its inhabitants have stepped back from use of nuclear power. Nowadays in a period of surging energy prices, worldwide interests in new and renewable energy sources increased. The fossil fuel depletion and greenhouse gas emissions become International crisis. Many countries began looking for renewable energy sources as energy savings are paramount That is why use of alternative energies like geothermal energy, has become a serious challenge. The geothermal energy is on the rise as an environment-friendly and semi-permanent energy system. By 1980's geothermal heat pumps (GHP) started developing and gaining popularity in order to reduce heating and cooling costs. Geothermal energy is clean and safe and today supplies less than $10 \%$ of world energy.Technically, an optimal heat transfer between the medium and the buried collectors, like ground source heat pumps (GSHP) or borehole heat exchangers $(\mathrm{BHE})$, is necessary, to exploit the efficiently

Chauchois A ${ }^{1,2}$, Derbal R. ${ }^{1}$, Defer D. ${ }^{1}$, Brachelet F. ${ }^{1}$

${ }^{1}$ Laboratoire de Génie Civil et géo-Environnement ( $\mathrm{GGCE}$ ), Université

d'Artois, Faculté des Sciences Appliquées, Technoparc Futura, 62400

Béthune,

France

${ }^{2}$ Geosystem laboratory- Civil, Environmental, and Architectural Engineering, Korea University, Anam-dong, Seongbuk-gu, Seoul, Korea line 2: Country Korea renewable energy of soil $[1,2,3]$. Therefor measuring moisture content is crucial with any soil processes, particularly those that occur near the soil surface.

In ground heat pump applications, deposition or extraction of thermal energy from the ground is accomplished by using a ground heat exchanger (GHE), whose operation induces simultaneous heat and moisture flow in the surrounding soil. Thus, the energy efficiency of buried systems is directly related to the soil moisture content, which impacts the heat transfer. Therefore, thorough knowledge of the intricate nature of soils related to coupled heat and moisture in the ground is essential to both the design and operation of ground heat pump systems. A few papers dealing with the influence of soil moisture conditions on ground heat pump performance have recently been published $[4,5]$. It was found that the soil moisture content was a dominant factor responsible for seasonal thermal conductivity variations. The effect of soil conditions and thermal conductivity on heat transfer in ground heat storage was also demonstrated. That is why it is necessary to know not only the thermal properties for the use of heat pumps from the ground, but also to measure ground water content. As the thermal properties and water content of a soil are closely related, it makes sense to study one by the other. In different other fields studies highlight the relation between moisture content and heat transfer $[6,7,8]$. For optimal exploitation of the studied systems, the medium moisture content needs to be determined.

The actual work is based on inverse heat transfer analysis. The study considers a multilayered homogenous configuration of a tested soil (sand). Four separated layers at different moisture 
contents are considered. They represent the vertical moisture content variation in the multilayer. The moisture content noted bellow $\omega$ or $\mathrm{p}$, is imposed constant parameter vertically and horizontally in each layer. The actual method aims to estimate the four mean moisture contents of the multilayer. It is based on the temperature measurement at different positions during a test (Fig.1). The theoretical and numerical studies are based on the heat transfer equation and the finite difference method associated to an optimization algorithm: the LevenbergMarquardt Algorithm (LMA). In one hand the method uses a simple experimental setup. In the other hand it considers the only heat transfer to estimate a mass transfer parameter. These are the major interesting points of the method. The method can be very useful in several application areas.

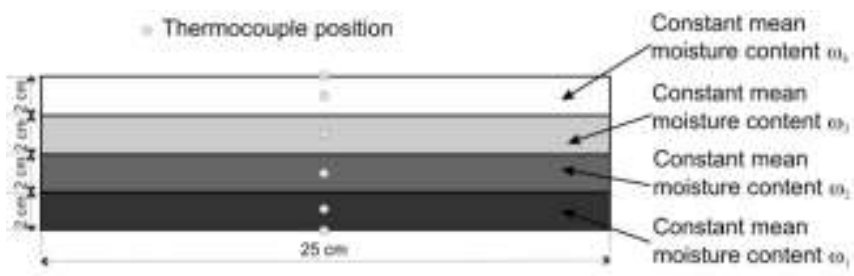

Figure 1. Sample tested in a multilayered configuration.

\section{Experimental setup}

\section{A. Material prior characterization:}

The moisture content obviously influences the heat transfer through a granular medium. It directly impacts the thermophysical properties of soils [9,10,11]. Relationships between the tested sand water content $(\omega)$ and its thermophysical properties $(\lambda$ : thermal conductivity and $\rho C$ : volumetric heat capacity) were established in previous works in our laboratory, and are used in this study [9] (Eq.1 and Eq.2). These empirical relationships are comparable to those cited in the literature $[12,13]$ (Table I).

$$
\begin{gathered}
\rho C(\omega)=-269.13 \cdot \omega^{2}+35.25 \cdot 10^{3} \cdot \omega \\
+130.67 \cdot 10^{4} \\
\lambda(\omega)=-8 \cdot 10^{-4} \cdot \omega^{2}+6.84 \cdot 10^{-2} \cdot \omega \\
+8.82 \cdot 10^{-2}
\end{gathered}
$$

The above Eq.1 and Eq.2 were defined for moisture contents greater than $5 \%(\omega \geq 5 \%)$. The devising of a method capable of linking the thermophysical quantities of the soil to its water content is therefore important, since these parameters appear to be closely interconnected.

TABLE I. THERMOPHYSICAL PROPERTIES ( 4 AND $C$ ) DEPENDING ON MOISTURE CONTENT $(\Omega)$

\begin{tabular}{|c|c|c|c|c|c|c|c|}
\hline$\omega(\%)$ & $\begin{array}{c}\mathbf{0 . 0 0} \\
\text { dry }\end{array}$ & $\mathbf{0 5 . 0}$ & $\mathbf{1 0 . 0}$ & $\mathbf{1 5 . 0}$ & $\mathbf{2 0 . 0}$ & $\mathbf{2 5 . 0}$ & $\begin{array}{c}\mathbf{3 1 . 7} \\
\text { saturated }\end{array}$ \\
\hline $\begin{array}{c}\lambda \_ \text {Chauchois } \\
\text { (W/K/m) }\end{array}$ & 0.25 & 1.20 & 1.50 & 1.70 & 1.90 & 2.10 & 2.20 \\
\hline $\begin{array}{c}\lambda \_ \text {Kersten } \\
\text { (W/K/m) }\end{array}$ & N/A & 1.23 & 1.56 & 1.76 & 1.89 & 2.00 & 2.12 \\
\hline $\begin{array}{c}C \_C h a u c h o i s \\
(\mathrm{~J} / \mathrm{K} / \mathrm{kg})\end{array}$ & 737 & 929 & 1058 & 1152 & 1249 & 1402 & 1593 \\
\hline
\end{tabular}

\begin{tabular}{|c|c|c|c|c|c|c|c|}
\hline $\begin{array}{c}C \_ \text {Laurent } \\
(\mathrm{J} / \mathrm{K} / \mathrm{kg})\end{array}$ & 770 & 898 & 1026 & 1154 & 1282 & 1409 & 1581 \\
\hline
\end{tabular}

Table II shows some characteristics of the used sand.

TABLE II. SAND PROPERTIES.

\begin{tabular}{|c|c|c|c|}
\hline Fine sand & $\begin{array}{c}\text { Fineness } \\
\text { Modulus }\left(\boldsymbol{M}_{\boldsymbol{f}}\right)\end{array}$ & $\begin{array}{c}\text { Dry density }\left(\gamma_{d}\right) \\
\left(\mathbf{k g} / \mathbf{m}^{\mathbf{3}}\right)\end{array}$ & $\begin{array}{c}\text { Moisture content at } \\
\text { saturation }\left(\boldsymbol{\omega}_{\text {sat }}\right)(\%)\end{array}$ \\
\hline$(0 / 0.45)$ & 1.2 & 1700 & 31.7 \\
\hline
\end{tabular}

\section{B. Experimental tests}

The experimental instrumentation consists of a tested multilayered material equipped with thermocouples, a flat heating resistance, a cold junction terminal block for differential measures, a data logger, and a control computer (Fig.2).

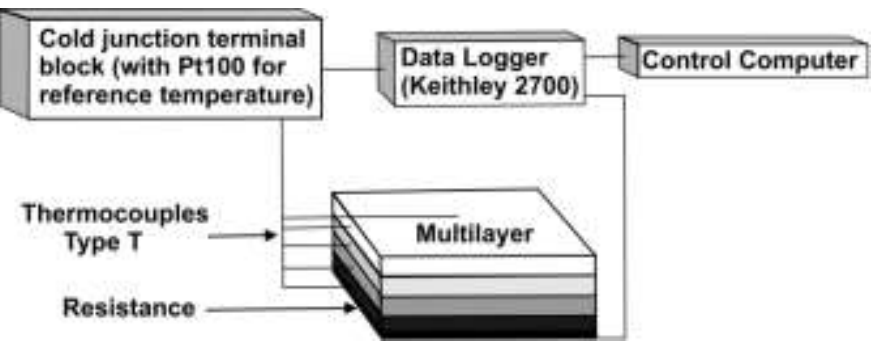

Figure 2. Experimental setup.

The multilayer is made of four thin layers of sand ( 2 $\mathrm{cm}$ thickness). Layers placed horizontally are at different moisture contents $\left(\omega_{1}, \omega_{2}, \omega_{3}, \omega_{4}\right) \approx(20 \%, 15 \%, 10 \%, 5 \%)$. These different moisture contents are achieved by wetting and mixing dry sand, after adding different amounts of water. During the sample preparation, the mean absolute error is estimated to be up to $0.5 \%$. The layers are arranged in decreasing order of moisture content, from the bottom to the top. Each layer is inserted in a $2 \mathrm{~cm}$ thickness polystyrene guard ring. This maintains sand, and limits lateral heat losses. Thus, a unidirectional heat transfer is guaranteed. Introduction of the sand in its guard ring is made in two parts. The first one is to fill in the first centimeter layer thickness without compaction on the whole surface $\left(25 \times 25 \mathrm{~cm}^{2}\right)$. The thermocouple is inserted in the middle of the layer. After that, the second centimeter layer thickness is filled, following exactly the same procedure as the first centimeter, with the same material and the same moisture content. This ensures correct positioning of the thermocouple centered in the middle of the layer volume $\left(25 \times 25 \times 2 \mathrm{~cm}^{3}\right)$. The same process is repeated for the three other layers. T-type thermocouples allow the temperature field to be recorded at the middle of each layer $\left(\mathbf{T}_{\text {meas } 1}, \mathbf{T}_{\text {meas } 2}, \mathbf{T}_{\text {meas 3 }}\right.$, and $\left.\mathbf{T}_{\text {meas } 4}\right)$. A thin (12 $\mu \mathrm{m}$ thickness) waterproof film is placed at the layers interfaces. This prevents layer water exchanges and losses, either liquid or vapor. Thus, it enables a maximum heat transfer, and a minimum mass transfer. Table III shows the layer mass 
evolution during a 12-hour test. The weight loss and moisture content deviation low values allows us to consider layers constant mean moisture contents. A thin film also separates the bottom layer, and the heating resistance $\left(25 \times 25 \mathrm{~cm}^{2}, 19.2\right.$ $\Omega$ ). The upper layer is in direct contact with the ambient air. These two last boundaries are equipped with T-type thermocouples in the middle surface at the top and the bottom. They allow us to record temperature fields at the extremities (boundary conditions: $\mathbf{T}_{\mathbf{u}}$ and $\mathbf{T}_{\mathbf{b}}$ ) (Fig.3).

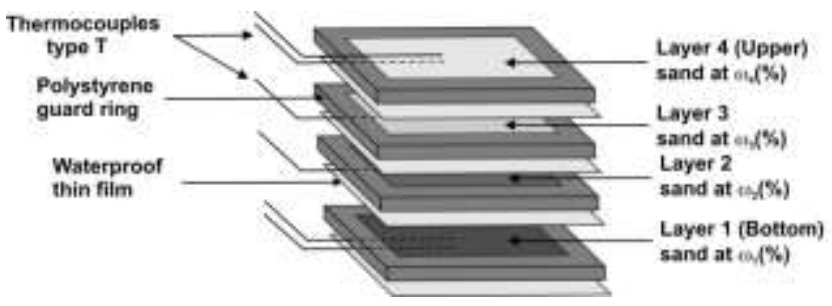

Figure 3. Tested multilayer details.

The heating resistance therefore bears all layers of the tested sample. It is placed on an insulating base, which directs the main heat flow dissipated by the resistance to the top, in the direction of the multilayer. This base consists of a Polyurethane plate $(5 \mathrm{~cm}$ thickness $)$ and PVC plate $(1 \mathrm{~cm}$ thickness), which offers a flat surface to the setup establishment. This experimental set-up configuration (polystyrene guard ring, thin waterproof film, heat source dimension equal to those of the samples ...) allows a unidirectional heat transfer. This was inspired by several standards, like ASTM C 1363-05 and ISO 8990. The thermocouples that are used are connected to a cold junction terminal block. A Pt100 resistance thermometer is also connected to the block, to provide a reference experimental temperature, which is necessary for differential measurement. The purpose of using a differential measurement terminal block is to overcome possible temperature fluctuations at the data logger card. The data logger is a multimeter scanner (Keithley 2700) fitted with a multiplexing card for measuring, and with two voltage output dedicated channels. One of the two channels stimulates the heating resistance via a power amplifier. A computer interface allows communication with the data logger and the power amplifier. It stores and prints the acquired data. The six measured temperature fields $\left(\mathbf{T}_{\mathbf{u}}, \mathbf{T}_{\text {meas }}\right.$ ${ }_{1}, \mathbf{T}_{\text {meas } 2}, \mathbf{T}_{\text {meas 3 }}, \mathbf{T}_{\text {meas 4 }}$, and $\mathbf{T}_{\mathbf{b}}$ ) during a test are numerically exploited.

TABLE III. LAYERS MASS EVOLUTION DURING ONE OF 12 HOUR TEST AT DIFFERENT MOISTURE CONTENTS.

\begin{tabular}{|c|c|c|c|c|}
\hline $\begin{array}{c}\text { Mean moisture content } \\
\text { values (\%) }\end{array}$ & 5 & 10 & 15 & 20 \\
\hline Mass before test (g) & 5312.4 & 5485.2 & 5750.1 & 6037.5 \\
\hline Mass after test (g) & 5308.1 & 5479.1 & 5735.3 & 6017.4 \\
\hline Weight loss (\%) & 0.07 & 0.11 & 0.26 & 0.33 \\
\hline $\begin{array}{c}\text { Absolute moisture } \\
\text { content deviation (\%) }\end{array}$ & 0.08 & 0.03 & 0.03 & 0.04 \\
\hline
\end{tabular}

\section{Numerical study}

\section{A. Numerical model}

Heat transfer occurs only through unidirectional conduction, without heat generation. This is due to the fact that the medium is supposed as solid, homogeneous and nontransparent. This is also due to the establishment of the experimental process, when for example, transversal sample's dimensions are greater than its thickness, or probes selected locations allow us to avoid edge effects (figures 1 and 4).

In this case, the heat transfer is determined by the heat conduction equation (Eq.3). One can connect it to the equations 1 and 2. . It is based on the finite difference method suitable for our case study [14].

$$
\frac{\partial T}{\partial t}=\frac{\lambda(\omega)}{\rho C(\omega)} \frac{\partial^{2} T}{\partial x^{2}}
$$

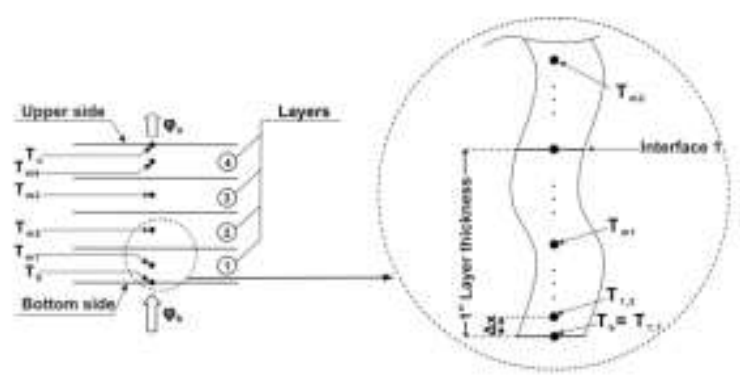

Figure 4. Tested multilayer and discretization details.

A multilayered horizontal material is studied (for the case of an in situ medium). The finite difference spatiotemporal discretization is applied to the multilayer. The successive four layers are discretized along the thickness into $M$ nodes $(j=0,1, \ldots, M)$. The space step between two nodes is $\Delta \mathrm{x}$. The total test time is discretized into $\mathrm{N}$ intervals $(\mathrm{i}=0$, $1, \ldots, N)$. The time step is $\Delta t=4 \mathrm{~s}$, which is also the temperature recording time step during an experimental test. The heat transfer is unidirectional (with no lateral loss, which was also confirmed by measuring soil temperature at positions other than the middle of the soil layer) through the thickness of the multilayer, where temperature evolution is simulated, after solving Eq. 3 as described below.

Numerically, we introduce the known initial condition, which is a one column matrix: $\mathrm{T}_{\mathrm{ini}}(j \Delta x)$. This is the initial temperature field through the multilayer thickness, carefully defined from initial recorded temperatures and noted $\mathbf{T}_{\text {ini. }}$. We also introduce the known two boundary conditions. Each boundary condition is a one column matrix representing the temperature field at the top: $\mathbf{T}_{\mathbf{u}}=\mathbf{T}_{\mathbf{u}}(i \Delta t)$, and at the bottom of the multilayer: $\mathbf{T}_{\mathbf{b}}=\mathbf{T}_{\mathbf{b}}(i \Delta t)$. These Dirichlet boundary conditions make it possible not to have to rely on the determination of the convection and radiation coefficient $h$. The matrix system of the finite difference method applied on the conductive heat transfer depends on the thermophysical 
properties and dimensions of the sample (four layers). Thus, it depends on the moisture content (four moisture content corresponding to each layer). Matlab ${ }^{\circledR}$ is used to solve the described numerical model, and thus provide all the simulated temperatures. Throughout the actual study, we are interested in the simulated temperatures in the middle of each layer (Fig.4): $\left(\mathrm{T}_{\mathrm{sim}}(\mathrm{m} 1, i \Delta t), \mathrm{T}_{\mathrm{sim}}(\mathrm{m} 2, i \Delta t), \mathrm{T}_{\mathrm{sim}}(\mathrm{m} 3, i \Delta t), \mathrm{T}_{\mathrm{sim}}(\mathrm{m} 4, i \Delta t)\right.$, where : $i=1, \ldots, \mathrm{N})$. For simplification, they are noted $\mathbf{T}_{\text {sim } \mathbf{1}}$, $\mathbf{T}_{\text {sim } 2}, \mathbf{T}_{\text {sim } 3}$ and $\mathbf{T}_{\operatorname{sim} 4}$, simultaneously.

The locations node $\mathrm{m} 1, \mathrm{~m} 2, \mathrm{~m} 3$ and $\mathrm{m} 4$ correspond to the nodes where the temperature fields are experimentally recorded: $\mathbf{T}_{\text {meas } 1}, \mathbf{T}_{\text {meas } 2}, \mathbf{T}_{\text {meas } 3}$ and $\mathbf{T}_{\text {meas } 4}$. Continuity at the interfaces is assumed to be established. The thin waterproof films placed between layers have a negligible thickness $(12 \mu \mathrm{m})$. Thus, their thermal résistance and heat capacity do not affect the conductive heat transfer [9]. Particular attention is paid when placing the films, so that additional contact resistances are avoided.

The results of the developed numerical model (simulated temperatures) based on the finite difference method and the results of experimental tests (measured temperatures) are both associated in a parametric estimation algorithm. This allows moisture content estimation of every layer, by fitting the simulated temperatures to the measured ones.

\section{B. Parametric estimation}

In this study we used the Levenberg-Marquardt algorithm (LMA) $[15,16]$. The connection between the experimental results, the finite difference numerical model, and the LMA leads to an inverse analysis model. The initial parameter set " $p_{0}$ " is a vector of four mean moisture content values (i.e. between $5 \%$ and $31.7 \%$ ). Numerically, other tested initial parameters provide similar results. Thermal properties are calculated from the parameter set "p" thanks to Eq.1 and 2. This allows to the numerical model to solve the heat equation (Eq.3) and to simulate temperature through the medium thickness. Then, the LMA compares them to the measured temperatures, and tries at each step to minimize an objective function that we defined, noted $\boldsymbol{F}$. In this work, the objective function $\boldsymbol{F}$ set from a weighted least square defined by the following equations 4 and 5 . This uses the four simulated and measured temperature vectors differences $\left(\boldsymbol{T}_{\text {meas } j}-\boldsymbol{T}_{\text {sim } j}, j\right.$ $=1, \ldots, 4)$. The LMA checks for the stopping criteria and then it either proposes another parameter set, or displays the last one found [17].

$$
\begin{gathered}
\boldsymbol{f}_{j}(\boldsymbol{p})=\frac{\boldsymbol{T}_{\text {meas } j}-\boldsymbol{T}_{\text {sim }}(\boldsymbol{p})}{\max \left(\boldsymbol{T}_{\text {meas } j}\right)-\min \left(\boldsymbol{T}_{\text {meas }}\right)} \\
\boldsymbol{F}(\boldsymbol{p})=\sum_{j}\left(\boldsymbol{f}_{j}(\boldsymbol{p})\right)^{2}
\end{gathered}
$$

Where: $j=1, \ldots, 4$ (nodes $\mathrm{m} 1, \ldots, \mathrm{m} 4)$ and $\mathrm{p}:$ vector of parameters to estimate (moisture content of each layer $\omega_{j}$ ).
At the end of the calculation, the algorithm outputs the last parameters set (four estimated moisture contents, one for each layer of the sample), as well as the simulated and measured temperature fields. The graphs offer information about the curve fitting and the objective function minimization.

In this work, numerical tests with the developed numerical model, demonstrate that Chauchois [9] empirical relationships (Eqs. 1 and 2) provide more precise results in comparison with the previous studies results[12,13]. This comes from the fact that in the actual study, we used exactly the same material used by Chauchois while Kersten established equations for ranges of soils. Concerning the specific heat, there is a significant agreement between the previous works of Chauchois and Laurent $[9,13]$. Using their results, we obtain the empirical relationships between the volumetric heat capacity and the moisture content. Therefore, equations 1 and 2 are used to represent the thermal behavior according to the water content.

From then on, we exploit the inverse heat transfer analysis for moisture content estimation to carry out a sensitivity study. This provides information about the reliability of the developed process and its settings, like the minimum necessary test time, or minimum valuable parameters.

\section{Results and discussion}

Previous studies allow us to define the relationships between the thermophysical properties $(\lambda$ and $\rho C$ ) and moisture content $(\omega)$. These results (Eqs. 1 and 2) were used in the current study. It is worth recalling that these relationships are only valid for $\omega \geq 5 \%$. These empirical relationships were introduced in the inverse heat transfer analysis. This allows the parametric estimation to deal with the four moisture contents of the tested layers, and not directly with their eight thermophysical properties. Thus, the optimization is improved, and the estimation errors are reduced.

A test campaign is a series of tests conducted on a sample using the experimental set-up. The test campaign aims to record the temperature fields at the middle of each layer of the sample and also at its ends (boundaries). From the bottom to the top of the multilayer, the measured temperatures are: $\mathbf{T}_{\mathbf{b}}$, $\mathbf{T}_{\text {meas 1 }}, \mathbf{T}_{\text {meas 2 }}, \mathbf{T}_{\text {meas 3 }}, \mathbf{T}_{\text {meas 4, }}$, and $\mathbf{T}_{\mathbf{u}}$. The thermal loads resulting from the flat heating resistance consist of randomly selected heat flux sequence levels. This allows the applicability of the device to be determined regardless of the excitation, and suggests in situ applications. The measurements are numerically exploited. This allows temperature simulation in the middle of each layer. Then, the LMA operates for parameter estimation.

The following figures and tables summarize the tests results. For clarity, the details of one test among others are presented on figure 5. Tables IV and V show the moisture 
content reference values $\left(\omega_{\text {ref }}\right)$, calculated moisture contents $\left(\omega_{\text {cal }}\right)$ and estimation errors.

TABLE IV. RESULTS OF ONE OF THE FOUR TESTS: STATISTICAL INDICATIONS ABOUT FIGURE 5 .

\begin{tabular}{|l|c|c|c|c|}
\hline Position & $\mathrm{m} 1$ & $\mathrm{~m} 2$ & $\mathrm{~m} 3$ & $\mathrm{~m} 4$ \\
\hline $\operatorname{Max}\left(\mathbf{T}_{\text {sim }}-\mathbf{T}_{\text {meas }}\right)\left(\mathbf{( 1 0}^{-2}{ }^{\circ} \mathbf{C}\right)$ & 10.5 & 5.9 & 8.3 & 7.7 \\
\hline Standard deviation $\left(\mathbf{1 0}^{-2}{ }^{\circ} \mathbf{C}\right)$ & 2.2 & 1.1 & 1.7 & 1.7 \\
\hline
\end{tabular}

TABLE V. RESULTS OF ONE OF THE FOUR TESTS: MOISTURE CONTENTS VALUES AND ESTIMATION ERRORS.

\begin{tabular}{cccccc}
\hline $\begin{array}{c}\text { Test Time } \\
\text { (Hours) }\end{array}$ & $\begin{array}{c}\text { Layer } \\
\text { number }\end{array}$ & $\begin{array}{c}\boldsymbol{\omega}_{\text {ref }} \\
(\boldsymbol{\%})\end{array}$ & $\begin{array}{c}\boldsymbol{\omega}_{\text {cal }} \\
(\boldsymbol{\%})\end{array}$ & $\begin{array}{c}\text { Absolute } \\
\text { Error }(\%)\end{array}$ & $\begin{array}{c}\text { Relative } \\
\text { Error }(\%)\end{array}$ \\
\hline & \begin{tabular}{c} 
(bottom) \\
\multirow{2}{*}{2.66}
\end{tabular} & 20.00 & 20.28 & 0.28 & 1.41 \\
& 2 & 15.00 & 16.38 & 1.38 & 9.18 \\
& 3 & 10.00 & 11.00 & 1.00 & 10.02 \\
& 4 (upper) & 5.00 & 5.08 & 0.08 & 1.66 \\
\hline
\end{tabular}

For a good parametric estimation, we take into account a minimum value of the moisture content, and a good initial temperature representation. Sequence levels of thermal load delivered by the heating resistance are used. The optimized time $(6000 \mathrm{~s})$ is learned from a performed sensitivity study (not presented here). Table VI summarizes the Tests series (4 tests were conducted, one after the other) results.

TABLE VI. TESTS RESUlts: MEAN VALUES OF THE FOUR TESTS.

\begin{tabular}{ccccc}
\hline Position & $\boldsymbol{\omega}_{\text {ref }}(\%)$ & $\omega_{\text {cal }}(\%)$ & $\begin{array}{c}\text { Absolute } \\
\text { Error }(\%)\end{array}$ & $\begin{array}{c}\text { Relative } \\
\text { Error }(\%)\end{array}$ \\
\hline $\mathrm{m} 1$ & 20.00 & 20.37 & 0.37 & 1.86 \\
$\mathrm{~m} 2$ & 15.00 & 15.80 & 0.80 & 5.35 \\
$\mathrm{~m} 3$ & 10.00 & 10.40 & 0.40 & 4.00 \\
$\mathrm{~m} 4$ & 5.00 & 4.40 & 0.60 & 12.05 \\
\hline
\end{tabular}

The results presented show a good parametric estimation, in light of the low relative error values. A good curve fitting is related to this parametric estimation. Table VI shows that when the moisture content is close to or below the minimum moisture content threshold ( $5 \%$ at position $\mathrm{m} 4$ ), the empirical relationships (Eqs. 1 and 2) are no longer representative of the moisture content evolution related to the thermophysical properties. In this case, the estimation of this parameter is more difficult. Thus, it is estimated with high relative errors (> $12 \%)$. We consider the estimation on the whole tests campaign to be satisfactory. The well measured and simulated temperature curves fitting provide an appreciation of the objective

function
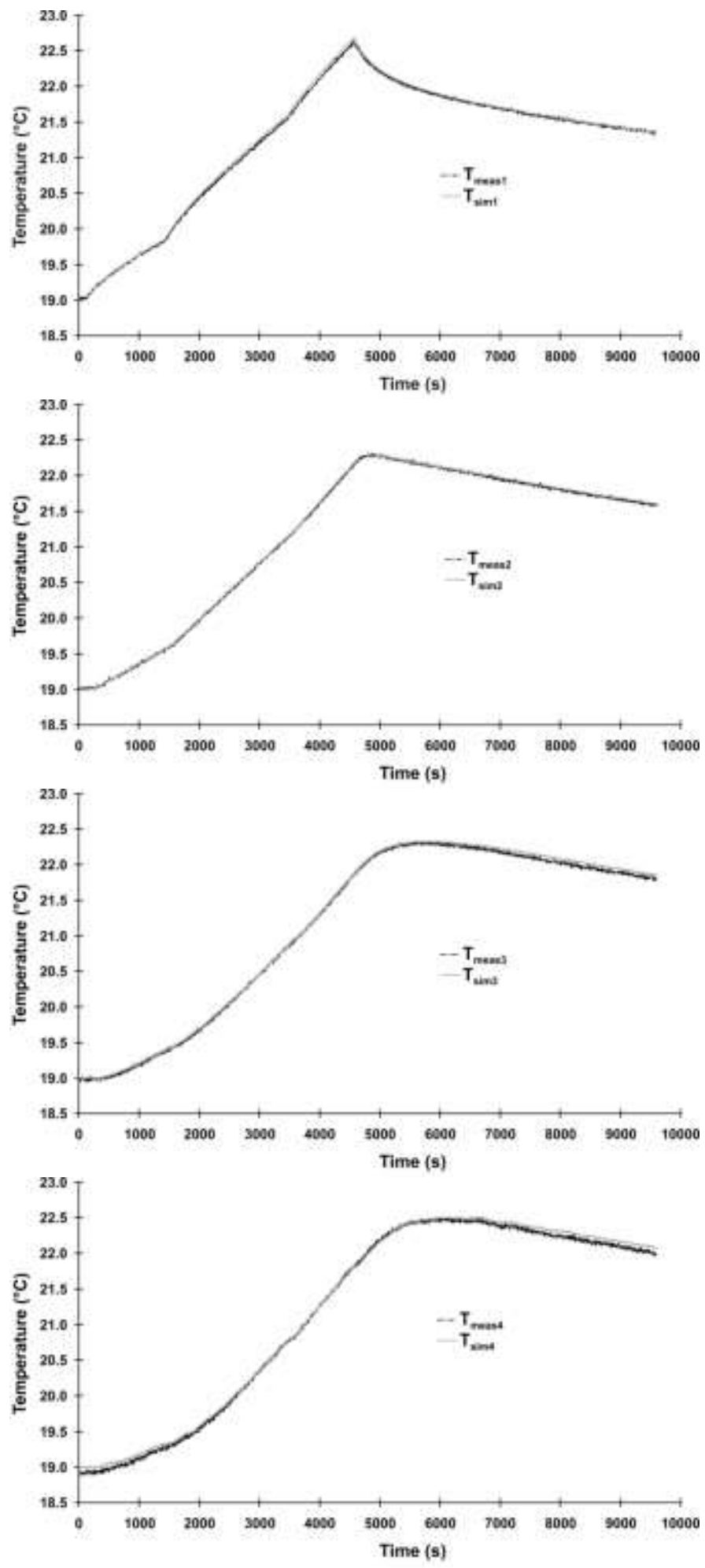

Figure 5. Results of one of the tests: Simulated after optimization and measured temperatures at the four nodes $\mathrm{m} 1, \mathrm{~m} 2, \mathrm{~m} 3, \mathrm{~m} 4$.

\section{v. Conclusion}

The article presents a conductive thermal diffusion analysis for moisture content characterization. This method is applied 
here on fine sand, which is a homogeneous, stable, thermally reversible, and easy to use.

First, to apply the method, we needed prior tests to determine the relationship between the thermophysical properties (thermal conductivity and volumetric heat capacity) and water content of the sand. Then, by an inverse heat transfer analysis through the tested sample, we estimate the different moisture contents. This method is applied to a multilayer of 4 sand layers at different moisture contents $(5 \%$, $10 \%, 15 \%$, and $20 \%$ ), separated by a thin waterproof film. The inversion algorithm implementation is based on a 1-D finite difference method. The algorithm exploits the temperatures measured by four thermocouples in the middle of each layer. The moisture contents were estimated with satisfactory relative errors of $1.86 \%$ to $5.35 \%$. For the driest $\left(\omega_{4} \approx 5 \%\right)$, the mean relative error is higher (i.e. 12\%). In this case, the correlation between thermophysical properties and moisture content reached its validity limit. In this work, we consider watertight layers. The moisture content is supposed to be uniformly distributed in the thickness of each layer. We now plan to study a multilayer with continuously varying moisture content. To do this, we can include in the heat transfer simulation algorithm a function describing the spatial (1-D) variations of the moisture content. The aim is to deal with moisture content monitoring, and reach an in situ stable application of the method.

\section{References}

[1] Lamarche, L., Kajl, S., Beauchamp, B., 2010. A review of methods to evaluate borehole thermal resistances in geothermal heat-pump systems. Geothermics, 39(2): 187-200.

[2] Lee, J.-Y., Hahn, J.-S., 2006. Characterization of groundwater temperature obtained from the Korean national groundwater monitoring stations: Implications for heat pumps. Journal of Hydrology, 329(3-4): 514-526.

[3] Molina-Giraldo, N., Bayer, P., Blum, P., 2011. Evaluating the influence of thermal dispersion on temperature plumes from geothermal systems using analytical solutions. International Journal of Thermal Sciences, 50(7): 1223-1231

[4] Diao, N., Li, Q., Fang, Z., 2004. Heat transfer in ground heat exchangers with groundwater advection. International Journal of Thermal Sciences, 43(12): 1203-1211.

[5] Garcia Gonzalez, R. et al., 2012. Interactions between the physical soil environment and a horizontal ground coupled heat pump, for a domestic site in the UK. Renewable Energy, 44(0): 141-153.

[6] Abdou, A., Budaiwi, I., 2013. The variation of thermal conductivity of fibrous insulation materials under different levels of moisture content. Construction and Building Materials, 43(0): 533-544.

[7] Antczak, E., Chauchois, A., Defer, D., Duthoit, B., 2003. Characterisation of the thermal effusivity of a partially saturated soil by the inverse method in the frequency domain. Applied Thermal Engineering, 23(12): 1525-1536.

[8] Dong, Y., Pamukcu, S., 2014. Thermal and electrical conduction in unsaturated sand controlled by surface wettability. Acta Geotech.: 1-9.

[9] Chauchois, A., 2002. Modèles d'ordres non entiers appliqués à la caractérisation thermique de milieux évolutifs par méthode inverse: cas d'un sol humide, Université d'Artois, Béthune, 217 pp.

[10] Kaemmerlen, A., Asllanaj, F., Sallée, H., Baillis, D., Jeandel, G., 2010. Transient modeling of combined conduction and radiation in wood fibre insulation and comparison with experimental data. International Journal of Thermal Sciences, 49(11): 2169-2176.

[11] Lipiec, J., Usowicz, B., Ferrero, A., 2007. Impact of soil compaction and wetness on thermal properties of sloping vineyard soil. International Journal of Heat and Mass Transfer, 50(19-20): 3837-3847.

[12] Kersten, M.S., 1949. Thermal Properties of Soils. Minn., University of Minnesota, $227 \mathrm{pp}$

[13] Laurent, J.P., 1986. Contribution à la caractérisation thermique des milieux poreux granulaires : Optimisation d'outils de mesure "in-situ" des paramètres thermiques. Application à l'étude des propriétés thermiques du matériau terre., Grenoble, $225 \mathrm{pp}$.

[14] Özişik, M.N., 1994. Finite difference methods in heat transfer. CRC Press, Boca Raton, Fla., 432 pp.

[15] Levenberg, K., 1944. A Method for the Solution of Certain Non-Linear Problems in Least Squares. Quarterly of Applied Mathematics, 2: 164168.

[16] Marquardt, D.W., 1953. An algorithm for least-squares estimation of nonlinear parameters. Journal of the Society for Industrial and Applied Mathematics, 11: 431-441.

[17] Derbal, R., Defer, D., Chauchois, A., Antczak, E., 2014. A simple method for building materials thermophysical properties estimation. Construction and Building Materials, 63(0): 197-205. 